\title{
Non invasive live cell cycle monitoring using quantitative phase imaging and proximal machine learning methods
}

\author{
Philippe Pognonec ${ }^{1}$, Michel Barlaud ${ }^{2}$, Benoit Wattellier ${ }^{3}$, Thierry Pourcher ${ }^{1}$, \\ Yuxiang Zhou ${ }^{2}$, Sherazade Aknoun ${ }^{3}$, Manuel Yonnet ${ }^{3}$, Marc Antonini ${ }^{2}$. \\ (1) TIRO, Faculté de Médecine, Univ. Côte d'Azur \& CEA, F-06107 Nice. \\ (2) I3S, Univ. Côte d'Azur \& CNRS, F-06900 Sophia Antipolis. \\ (3) Phasics, Route de l'Orme des Merisiers, F-91190 Saint Aubin \\ Contact: barlaud@i3s.unice.fr
}

\begin{abstract}
This interdisciplinary work focuses on the interest of a new proximal algorithm (ADRS ) for supervised classification of live cell populations growing in a thermostated imaging station and acquired by a Quantitative Phase Imaging $(Q P I)$ camera. This type of camera produces interferograms that have to be processed in order to extract features derived from quantitative linear retardance and birefringence measurements. We monitor cell cycling in different populations with both a classical fluorescent DNA intercalating agent imaging on the one hand and $Q P I$ without any cellular manipulation nor treatment on the other hand. We show that the accuracy of the classification of these cells in different phases of the cell cycle is equivalent, if not better, when using $Q P I$ features as compared to fluorescence imaging features. This is a very important finding since we demonstrate that it is now possible to very precisely follow cell growth under regular culture conditions without any bias. No dye or any kind of markers are necessary for this live monitoring, thus the cells normal physiology is not at all affected by this non invasive procedure. Any studies requiring analysis of cell growth or cellular response to any kind of treatment could benefit from this new approach.
\end{abstract}

\section{PRoblem STATEMENT}

Cell cycle is a biological process ultimately resulting in cell division, yielding two daughter cells with the same exact genetic content. This highly regulated process is of the utmost importance and is a direct reflection of cell growth. It consists of four phases:

i) the mitotic phase per se (M-Phase), in which nuclei lose their membrane, nucleoli disappear and chromosomes are physically sorted to be equally split among two identical daughter cells. These new cells then enter:

ii) G1 phase, in which cell nucleus gets reorganized following cell division. The entry into G1 phase requires the passage of a checkpoint ("Spindle checkpoint") where cells verify that division occurred as planned. If not, cells cannot proceed to G1. G1 phase is associated with the formation of nucleoli, sub-nuclear structures that produce ribosomal RNAs required for the synthesis of proteins from transcribed DNA, as well as for the normal physiology of the cell. DNA transcription into RNA restarts in order to support cellular functions. Following G1 phase and after another checkpoint validating their well being, the cells proceed to:

iii) $\mathrm{S}$ phase, where DNA is replicated. This phase is associated with an increase in cell size and mass. After chromosomes have been duplicated, cells enter:

iiii) G2 phase, where there is a last checkpoint before entering M-Phase again.

While monitoring cell cycling allows to directly follow cell growth, no method is currently available to do so without interfering with normal cell growth. Indeed, either FluorescenceActivated Cell-Sorting (FACS) that requires non-physiological cell handling or cell labelling with exogenously expressed fluorescent proteins are used. We investigated whether highresolution quadri-wave lateral shearing interferometry, that allows quantitative linear retardance and birefringence measurements on biological samples could allow non invasive monitoring of cell cycle in populations. This Quantitative Phase Imaging (QPI) strategy, that has been presented in [19, 6], only requires short bursts of low intensity white light, whose effect on cell physiology appears negligible. The optical phase difference measured when photons exit a biological sample is a direct reflection of the dry mass present in the sample being analyzed. It has been shown for example that the mass increase associated with cellular cell cycle progression ( $2 \mathrm{~N}$ toward $4 \mathrm{~N}$ chromosomes) can be accurately determined [1]. In addition, the characteristic cell rounding associated to late G2 phase is also easily detected by QPI.

Biologists partners investigated how reliable this approach would be to monitor a live cell population under the microscope. They first setup a controlled system in which HeLa cells were synchronized in early $\mathrm{S}$ phase (double thymidine block) and late G2/mitosis (nocodazole block) [16]. Cells were classically labeled with Hoechst 33342 in order to visualize nuclei by fluorescence of the dye. Multiple fields were then acquired in parallel with a regular CMOS camera (Zyla 5.5) to visualize the blue Hoechst fluorescence, and with a SID4Bio camera in order to acquire quantitative phase images. The goals are the following:

- To identify a feature footprint (phase and fluorescence) for each subtype of cells (control, "nocodazole" ,"thymidine"). 
- To figure out if phase footprint only can predict automatically the subtype of cells.

In the next section, we provide a robust classification method to identify these different populations from features extracted from QPI acquisitions.

\section{SUPERVISED CLASSIFICATION AND FEATURE SELECTION}

\section{A. Modelization}

In this paper we consider methods where feature selection is embedded in a classification process [14]. It is well-known that classification in high dimension suffers from the curse of dimensionality. In order to overcome this issue, the main idea of the following methods is to project data in a low dimensional space. A popular approach for selecting sparse features in supervised classification is the Least Absolute Shrinkage and Selection Operator (LASSO). formulation [13]. However, an issue is that using an $\ell_{2}$ norm for the data is not robust to outliers. In this paper, we cope with this problem by minimizing an $\ell_{1}$ norm both on the penalty term and the loss function. In this case, the criterion is convex but not gradient Lipschitz. Thus, we propose to use the Douglas-Rachford splitting method for the minimization of our criterion. This splitting was successfully used in signal processing $[10,8]$. However, for classification, we cannot apply straightforwardly Douglas-Rachford since the proximal operator for the affine transform $Y-X W$ involved in the criterion is not available. We present in the next sections the two approaches: minimizing the $\ell_{2}$ norm and then minimizing the $\ell_{1}$ norm.

Let $X$ be the $m \times d$ matrix made of $m$ line cells $x_{1}, \ldots, x_{m}$ belonging to the $d$-dimensional space of features. Let $Y \in\{0,1\}^{m \times k}$ be the label matrix where $k \geqslant 2$ is the number of clusters. Each line of $Y$ has exactly one nonzero element equal to one, $y_{i j}=1$ indicating that the sample $x_{i}$ belongs to the $j$-th cluster. Let $W \in \mathbb{R}^{d \times k}$ be the projection matrix, $k \ll d$. The basic idea is to project data on a low dimensional space $X * W$ with a strict control on the sparsity of $\mathrm{W}$. The goal is to compute the projection matrix, $W \in \mathbb{R}^{d \times k}$.

\section{B. Minimizing the Frobenius norm}

This result in an optimization problem minimizing the within sum of squares in the clusters (Frobenius norm) with an $\ell_{1}$ penalty in order to promote sparsity: a method called LASSO (Least Absolute Shrinkage and Selection Operator).

$$
\min _{W} \frac{1}{2}\|Y-X W\|_{F}^{2}+\lambda\|W\|_{1}
$$

To solve this problem, we use a gradient-projection method. It belongs to the class of proximal splitting methods [17, 10,4] using separately the convexity properties of the Frobenius cost on one hand, and of the convex constraint $\|W\|_{1}$ on the other. We use the following forward-backward scheme to generate a sequence of iterates for any fixed step $\gamma \in\left(0,2 / \sigma_{\max }^{2}(X)\right)$.

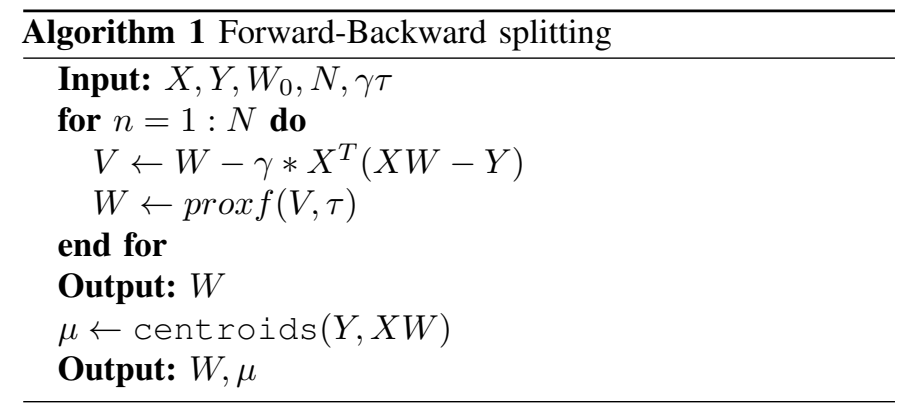

where $\operatorname{proxf}(V, \tau)$ denotes the proximity operator of the $\ell_{1}$ norm (soft thresholding) [10]

$$
\begin{aligned}
\operatorname{soft}(x, \tau) & =x+\tau \text { if } x<-\tau, \\
& =0 \text { if } x \in[-\tau, \tau] \\
& =x-\tau \text { otherwise. }
\end{aligned}
$$

\section{Minimizing the $\ell_{1}$ norm}

It is well known that the quadratic Frobenius loss criterion is not robust to outliers, so we propose to minimize instead the $\ell_{1}$ loss cost, with an $\ell_{1}$ penalty regularization to promote sparsity and induce feature selection. So, given the matrix of labels $Y$, we consider the following convex supervised classification problem:

$$
\min _{W}\|Y-X W\|_{1}+\lambda\|W\|_{1}
$$

The cost is the sum of two $\ell_{1}$ norms, which suggests to use a Douglas-Rachford splitting method [11, 10,9] that is able to cope with mere convex functions, one still has to be able to compute their proximity operators. Now, while the prox of the $\ell_{1}$ norm is well known and expressed in terms of soft thresholding, there is no explicit expression for the prox of the $\ell_{1}$ norm of the affine transform $Y-X W$. We propose to introduce the auxiliary variable ( see [5] for more details) $\zeta:=(Y-X W) / \lambda$ in $\mathbf{R}^{m \times k}$ and to minimize under the affine constraint:

$$
\begin{array}{r}
\min _{W}\|W\|_{1}+\|\zeta\|_{1} \\
X W+\lambda \zeta=Y .
\end{array}
$$

The sum of the two $\ell_{1}$ norms is equal to the single $\ell_{1}$ norm of the augmented variable $\tilde{W}:=(W, \zeta) \in \mathbf{R}^{(d+m) \times k}$. Let $C$ be the affine subset of $(d+m) \times k$ matrices such that $\tilde{X} \tilde{W}=Y$ where $\tilde{X}:=\left[\begin{array}{ll}X \lambda I_{m}\end{array}\right] \in \mathbf{R}^{m \times(d+m)}$. The problem can be recast as a minimization under the affine constraint

$$
\begin{gathered}
\min _{W}\|\tilde{W}\|_{1} \\
\tilde{X} \tilde{W}=Y
\end{gathered}
$$

Thus we get the following algorithm 


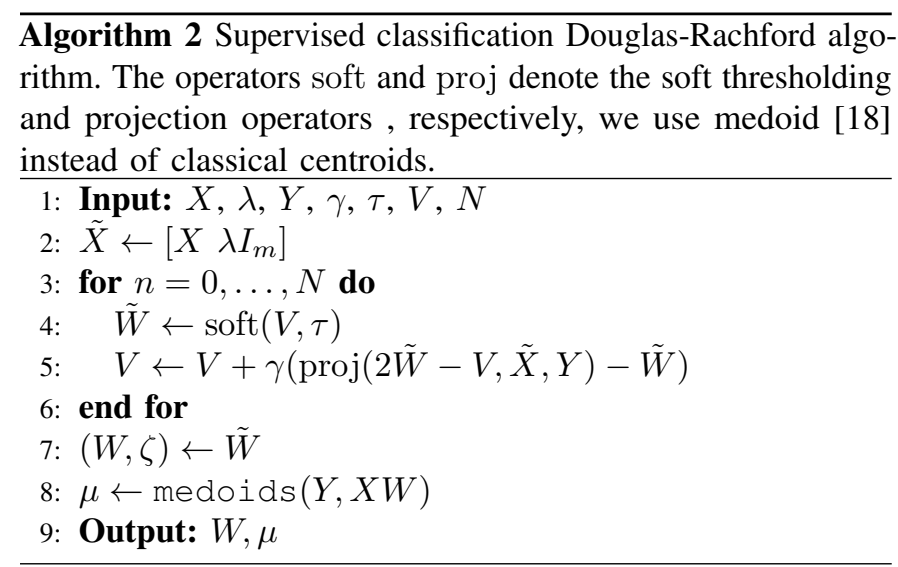

Definition of proj: Let $A$ be an $m \times n$ matrix of rank $m<n$, and let $b$ be a vector in $\mathbf{R}^{m}$. The orthogonal projection proj of $z \in \mathbf{R}^{n}$ on the subspace on the affine subspace $\{x \in$ $\left.\mathbf{R}^{n} \mid A x=b\right\}$ is

$$
\operatorname{proj}(z, A, b)=z-A^{T}\left(A A^{T}\right)^{-1}(A z-b) .
$$

Feature selection is achieved by means of the $\ell_{1}$ sparsity inducing penalty cost. A feature $i \in\{1, \ldots, m\}$ is then selected if the corresponding line in the matrix of weights $W$ is not zero $(\|W(i,:)\| \neq 0)$. The set of non zero coefficients of matrix $\mathrm{W}$ is the signature of the process.

\section{Quantitative Phase Imaging}

In this paper, we focus on the interest to use quantitative phase imaging to make high content screening experiments.

\section{A. Optical Path Difference}

When light propagates through a sample, its interaction with matter creates local delays due to the change of its velocity by a factor named refractive index. The accumulated delay relates to the phase of the light complex electromagnetic field:

$$
A(\vec{r})=\sqrt{I(\vec{r})} e^{j \varphi(\vec{r})}
$$

where $A$ is the field amplitude, $I$ the field intensity and $\varphi$ the field phase. In the projective approximation, where the field depth is much larger than the sample thickness, the field phase is related to the refractive index field $n$ by

$$
\varphi(\vec{r})=\frac{2 \pi}{\lambda} \int n(\vec{r}, z) d z
$$

where $z$ is the propagation direction. Phase is often identified to the Optical Path Difference $W$ (OPD), independent upon the wavelength defined as:

$$
W=\frac{\lambda}{2 \pi} \varphi=\int n d z
$$

\section{B. Phase Imaging with a wave front sensor}

The phase field is not easily revealed since standard cameras are only sensitive to the field intensity. Zernike first proposed to create contrast from phase information and DIC or Nomarski imaging is now widely implemented on microscopes. However the phase value is not easily retrieved from these techniques.
Digital holography $[12,15]$ was the first method to obtain Quantitative Phase Images (QPI) in microscopy. In this paper, we propose to use a wave front sensor to produce QPI images. A wave front sensor directly measures the phase and the intensity of imaged field. Using a wave front sensor for QPI makes the implementation on a microscope straightforward since it replaces a standard video camera at any of exit port of a microscope and is compatible with any illumination means. The wave front sensor used for these experiments is based on QuadriWave Lateral Shearing Interferometry (QLSI) [19, 6] and is developed by PHASICS (SID4 Bio). This technique improves the definition (ie the number of measurement points per camera pixels) of standard wave front sensing techniques like Shack-Hartmann.

The incident light field is sampled by a diffraction grating, which generates four tilted replicas of the field (see Fig. 1). They interfere on a camera chip placed a few millimeters downstream. The recorded camera image is a pattern made of a deformed grid which fundamental period is the same as the diffraction grating period $p$.

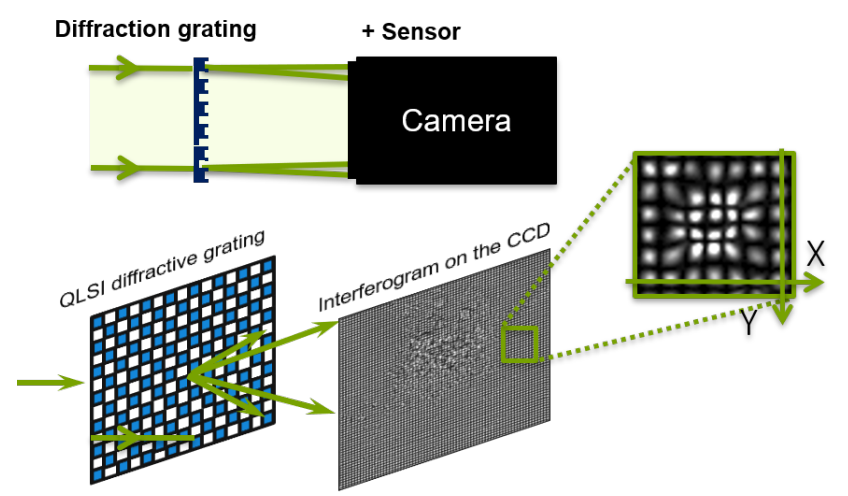

Fig. 1: Illustration of the QLSI principle. Top : Side view of the interferometer. The light impinging from the left diffracts through a diffraction grating and then interferes on the camera detector. Bottom : perspective view of the interferomter. The diffraction grating is made from the Modified Hartmann Mask principle, where a grid of holes is phase modulated by $\pi$ every other hole. Interference fringes (here two interference patterns cross which leads to dot-like fringes) are deformed by phase gradients.

It was shown [6] that the grid deformation is proportional to the OPD spatial derivative $\frac{\partial W}{\partial x}$. The interference field $I_{\text {int }}$ is given by:

$$
I_{\text {int }}(x)=I(x)\left[1+\cos \left(\frac{2 \pi}{p} x+\frac{2 \pi z}{p} \frac{\partial W}{\partial x}\right)\right]
$$

where $z$ is the distance between the grating and the camera chip. Here is the equation is given in 1D for sack of simplicity whereas it is easily to $2 \mathrm{D}$. The OPD gradient field is recovered from Fourier filtering around the fundamental frequency $\frac{1}{p}$ and finally numerically integrated. As seen in the equation above, the inteferogram field is independent of the wavelength which makes this techniques achromatic, which means it is compatible with white light and LED illumination. 


\section{QPI features}

To feed the classification with QPI features, phase images containing cells are first segmented. Then every cell is measured to determine morphological and quantitative features (see Fig. 2). Morphological features are those commonly used for white light or fluorescence images. They include cell surface, ellipticity, circularity, convexity and solidity.

Quantitative features are analog to radiometric features and are related with the pixel values. It is important here to mention that pixel values are rather different from gray level values. The latter are only to be considered relatively, whereas quantitative phase imaging values are calibrated and relate to physical parameters, namely the local mass density (mass per unit of surface). Therefore values are consistent from one image from another. In this study, we used 6 QPI features : dry-mass, average density, Max OPD, Mean OPD, OPD Median, OPD standard deviation. Other features describing mass inhomogeneities (texture features for instance) could also be included.

We here mentioned dry-mass and density which not direct OPD features. However it was shown by [3] that the mass volume density $\rho$ is proportional to the refractive index $n$ :

$$
n=n_{\text {medium }}+\alpha \rho
$$

It was also shown that $\alpha$ is almost constant for any intracellular components (lipids, proteins, DNA...). Since the OPD is the integral of the refractive in one direction, it is proportional to the mass surface density. Therefore the average OPD inside the cell is rescaled by a factor $\alpha$ and is the identified to the average mass surface density:

$$
\alpha \simeq 0.19 \mathrm{pg} / \mu \mathrm{m}^{3}
$$

Similarly if the OPD is integrated over the cell surface, we obtain a figure proportional to the call dry-mass. The cell drymass is the mass of the cell excluding water, which is the surrounding medium.

$$
m=\iiint \rho=\frac{1}{\alpha} \iint O P D
$$

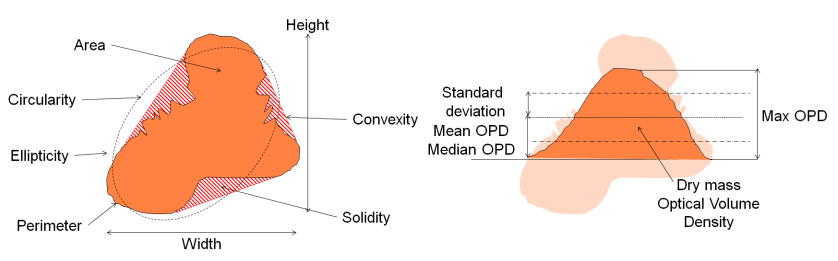

Fig. 2: Features extracted from QPI images of segmented cells. Left : Morphological features as defined in CellProfiler software [7]. Right : QPI features based on QPI image pixel values.

\section{EXPERIMENTAL RESULTS}

First, both visible (fluorescent labeling) and phase images were processed with software (CellProfiler for fluorescent images [7], and BioData (Phasics) for QPI to extract multiple features stored as matrix (the $\mathrm{X}$ matrix defined in section II), in which each line corresponds to a detected cell/nuclei and each column displays the values of a specific feature (diameter, phase value, ...) for all the cells. In our experiment we have $d=77$ features: 64 features for fluorescent images and 13 features for QPI images. The following work was done on the widely used HeLa cells, an immortalized line derived in 1953 from an epidermoid carcinoma of the cervix [21]. The results presented here remain valid on other cell types (data not shown). Hoechst 33342 labeling allows nuclei visualization by fluorescence, and to clearly detect in a proliferating population mitotic structures where chromosome condensation reaches a peak (Fig. 3, Panel A, arrows). Interferogram processing of the same field acquired via QPI produces the image shown in Panel B, where cells have been segmented. One can clearly see that the two mitotic cells visualized by nuclear staining correspond to cells with a high density signal, as pointed by the two red arrows. In addition to the mitotic events, a third cell (green and red dots) also present a higher density reflecting a higher dry mass, thus a higher DNA content. This cell is likely in late G2.

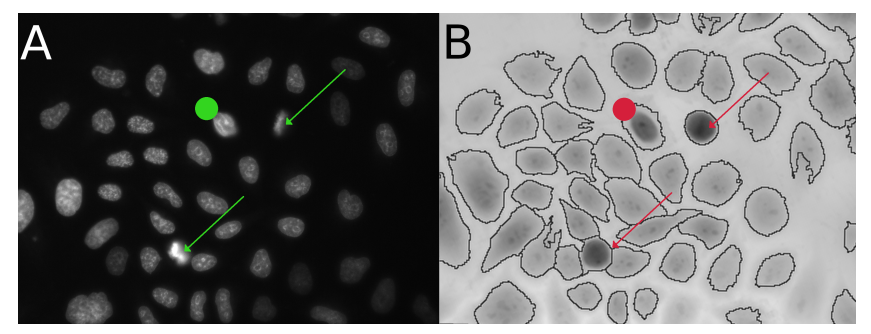

Fig. 3: "Fluorescence versus Phase imaging", Panel A: Fluorescence labeling of nuclei, Panel B: Phase imaging. The arrows point to cells in $\mathrm{M}$ phase, clearly visualized both in fluorescence and phase imaging. The equatorial plate, corresponding to chromosome alignment during metaphase can be seen in phase imaging in the upper right pointed cell. Big dots point to a cell not particularly different from the other ones in fluorescence imaging, but which is clearly denser in phase imaging, indicating a cell with duplicated chromosomes close to entering mitosis.

There are two approaches to evaluate the contribution of the features. The first one considers classical dotplots using two specific features (see Fig. 4). The second machine learning approach uses the matrix $\mathrm{W}$ as signature (a linear combination of features) (see Fig. 5). Thus we can plot the data in the projected space $(k \times k)$ using signature rather than considering sets of two specific features.

In a first classical approach, we took advantage of the powerful topcat application [22] to obtain dotplots in which $\mathrm{x}$ and $\mathrm{y}$ axes were chosen among the features available for both fluorescence and phase images. This demonstrated that cells arrested in mitosis by Nocodazole block or early $\mathrm{S}$ by Thymidine treatment form homogeneous populations. More interestingly yet, we found that features from $Q P I$ alone were very effective in separating the different populations (see Fig. 4). As expected, some of the cells from the control population presented feature values similar to those of the 2 blocked populations, since there are cells in early G1 as well as cells in mitosis in the control. 


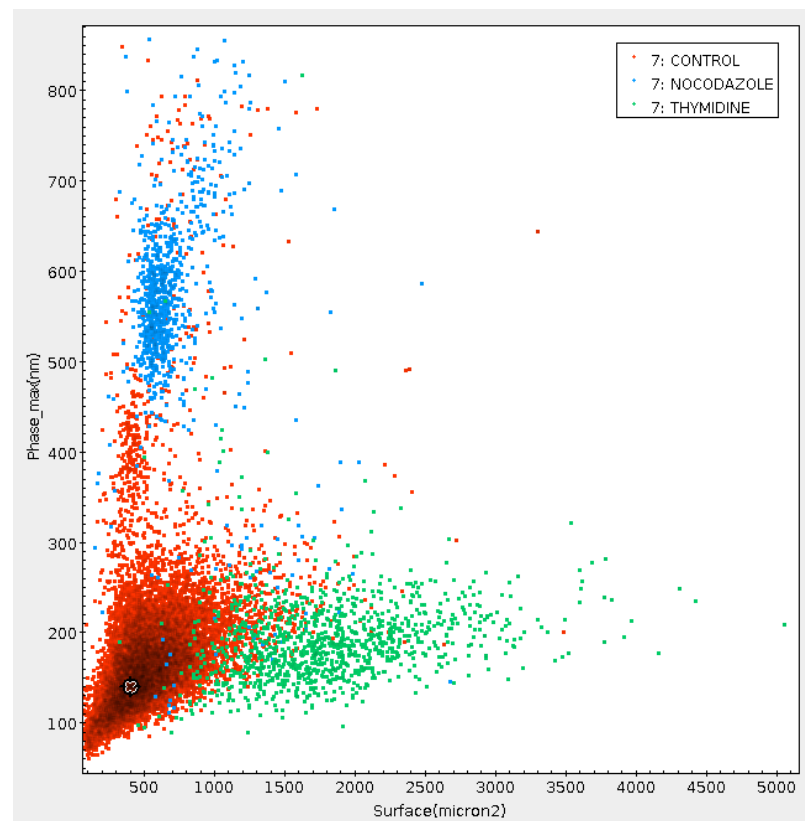

Fig. 4: "Dot-plot visualization of Surface versus Phase Max QPI features of mixed Control+Thymidine+Nocodazole cell populations". Red: Control cells; Green: thymidine treated cells: Blue: nocodazole treated cells. This dotplot demonstrates that Phase features allow the separation of the different populations according to their localization in the cell cycle. Interestingly, $\mathbf{M}$ phase cells (blue) form a characteristic sub-population, allowing for an accurate calculation of the proportion of cells in mitosis, i.e. the determination of the proliferation status of the cell population monitored.

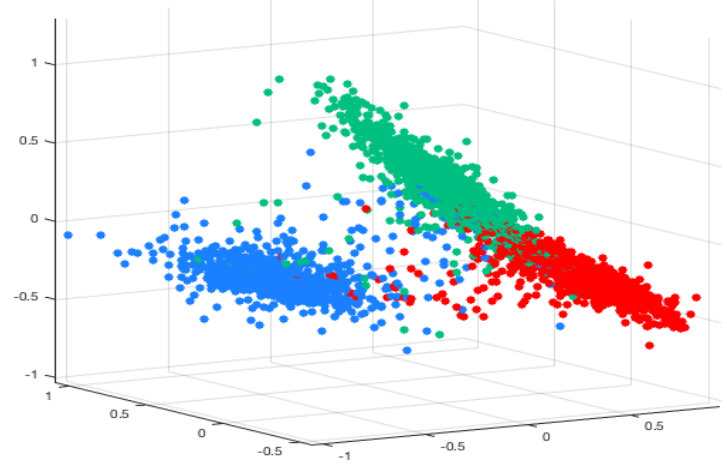

Fig. 5: The machine learning approach uses the matrix $\mathrm{W}$ in the projected $k \times k$ space as signature. It is a global signature evaluation as opposed to the pair wise topcat approach. This figure shows that the same data as that presented in Fig. 4 is accurately clustered in the projected space. Red: Control cells; Green: Thymidine treated cells; Blue: Nocodazole-treated cells.

Very interestingly, we observed that not only did our ADRS classification method gives very high accuracy results when performed on all available features from both fluorescent imaging and QPI analyses, but also that this accuracy was at

\begin{tabular}{|c|c|c|c|c|}
\hline Accuracy & Mean & Control & Noco & Thym \\
\hline ADRS all features & 92.97 & 93.69 & 93.23 & 91.68 \\
\hline Lasso all features & 91.4 & 94.09 & 90.81 & 89.53 \\
\hline \hline ADRS QPI only & 91.86 & 95.99 & 91.83 & 88.34 \\
\hline ADRS fluo only & 89.24 & 87.66 & 89.90 & 90.05 \\
\hline
\end{tabular}

TABLE I: Accuracy with Phasics + Fluorescence features with Zscore normalization. 3000 cells (sampling on control), 77 features and $k=3$ clusters. According to matrix $w$ the 4 main phase discriminant features are:

Phase-avg(nm),

Surface-density(pg/micron2),

Surface(micron2),

Phase-median(nm).

least as good when only Phase features were used, as compared to fluorescent only features (see Table I). This indicates that analysis of QPI is at least as efficient as classical fluorescent imaging in extracting features that are characteristic of cell positions in their cycles. Phenotypic comparison performed with topcat visualization tool demonstrated these similarities (see Fig. 6).

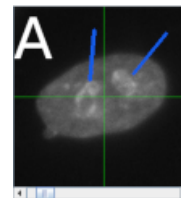

B

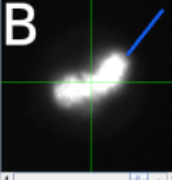

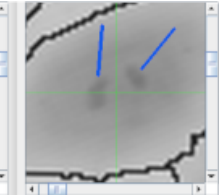

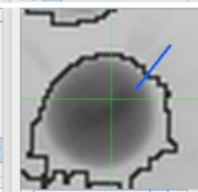

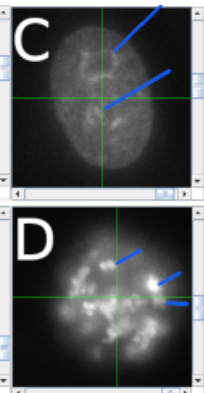

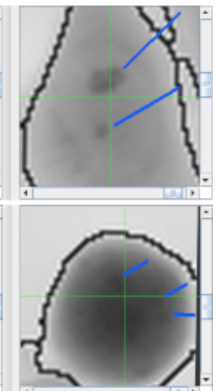

Fig. 6: "Fluorescence versus Phase imaging, details", Panel A: Fluorescence (left) and phase (right) imaging of a control cell in $\mathrm{S}$ phase, similar to the one seen in Panel $\mathrm{C}$ below. Nucleoli (blue lines) are clearly visible in fluorescence, and also readily visible with phase imaging. Panel B: This control cell is in metaphase (second stage of mitosis), and is not very different from the nocodazole-blocked cell seen in Panel D. The blue line points to the equatorial phase (chromosome alignment during metaphase), which is also distinguishable with phase imaging. Panel C: Cell blocked with thymidine in early $\mathrm{S}$ phase, with clearly visible nucleoli (blue lines). Panel D: Cell blocked during mitosis with nocodazole. The blue lines point to a few of the chromosomes stuck by nocodazole in prophase, the first stage of mitosis. Chromosome visualization in phase imaging is possible but difficult as compared to fluorescence imaging (where labelling is chromosome specific). However, the phase analysis of the image unambiguously indicates a bona fide mitotic cell.

\section{CONCLUSion}

This work demonstrates that Quantitative Phase Imaging (QPI) is a very powerful and non invasive way for biologists to monitor live cell populations with a relatively standard 
imaging station consisting of an automated microscope in a $\mathrm{CO} 2$ thermostated and water-saturated incubation chamber. Classically, biologists interested in following cell populations under live conditions have to rely on either "vital" labeling, such as the DNA intercalating agent Hoechst 33342, or exogenously expressed proteins tagged with a fluorescent domain. However, it is well known that "vital" dyes are quick to alter cell physiology, as seen with Hoechst 33342 mentioned above [20]. Similarly, while very useful, fluorescent fusion proteins result in many possible artifacts and toxicity, and thus cannot be considered as physiological [2] The camera used in this study does not capture classical photonic images, but rather records interferograms of the live cells by measuring regular white light phase gradients created when going through the biological samples. From these interferograms, several cell features are extracted. Mathematical treatment of these features allows for a very accurate supervised classification of existing sub-populations. This means that without any treatment to the cells, biologists are now able to calculate the proportion of cells going through mitosis, in a way reminiscent of the widely used FACS systems. But contrarily to FACS analyses, the cells remain untouched in culture during the whole process, that can theoretically last as long as needed. In other words, all questions dealing with cell growth and phenotypic changes in cell populations can now be addressed in real time, and evolution in time of the population is accessible. A straightforward application, among others, would be live real time pharmaceutical screening of molecule banks.

\section{ACKNOWLEDGEMENTS}

This work has been supported by the French Agence National de la Recherche (ANR), through the ANR-14-CE17-0017 grant.

\section{REFERENCES}

[1] S. Aknoun, P. Bon, J. Savatier, B. Wattellier, and S. Monneret. Quantitative retardance imaging of biological samples using quadriwave lateral shearing interferometry. Optics Express, 23 (12):16383, June 2015.

[2] A. M. Ansari, A. K. Ahmed, A. E. Matsangos, F. Lay, L. J. Born, G. Marti, J. W. Harmon, and Z. Sun. Cellular GFP Toxicity and Immunogenicity: Potential Confounders in in Vivo Cell Tracking Experiments. Stem Cell Reviews, 12:553-559, 2016.

[3] R. Barer. Inteference microscopy and mass determination. Nature, 169:366-367, 1952.

[4] M. Barlaud, W. Belhajali, P. L. Combettes, and L. Fillatre. Classification and regression using an outer approximation projection-gradient method. IEEE Transactions on Signal Processing, 65(17):4635-4644, 2017.

[5] M. Barlaud, Y. Zhou, J. Caillau, and M. Antonini. Robust Classification with feature selection using alternating minimization and Douglas-Rachford Splitting Method. ArXiv e-prints, (2368515), August 2018.

[6] P. Bon, G. Maucort, B. Wattellier, and S. Monneret. Quadriwave lateral shearing interferometry for quantitative phase microscopy of living cells. Opt. Express, 17:13080-13094, 2009.

[7] A. E. Carpenter, T. R. Jones, M. R. Lamprecht, C. Clarke, I. H. Kang, O. Friman, D. A. Guertin, J. H. Chang, R. A. Lindquist, J. Moffat, P. Golland, and D. M. Sabatini. CellProfiler: image analysis software for identifying and quantifying cell phenotypes. Genome Biology, 7(10):R100, 2006.

[8] A. Chambolle and T. Pock. A first-order primal-dual algorithm for convex problems with applications to imaging. Journal of Mathematical Imaging and Vision, 40(1):120-145, May 2011.
[9] J.-C. Combettes, P.L.and Pesquet. A douglas-rachford splitting approach to nonsmooth convex variational signal recovery. IEEE J. Selected Topics Signal Process., pages 564-574, 2007.

[10] P. L. Combettes and J.-C. Pesquet. Proximal splitting methods in signal processing. In Fixed-point algorithms for inverse problems in science and engineering, pages 185-212. Springer, 2011.

[11] J. Douglas and H. H. Rachford. On the numerical solution of heat conduction problems in two or three space variables. Trans. Amer. Math. Soc., pages 421-439, 1956.

[12] F. B. E. Cuche and C. Depeursinge. Digital holography for quantitative phase-contrast imaging. Opt. Lett., 47(5):291-293, 1999.

[13] J. Friedman, T. Hastie, and R. Tibshirani. Regularization path for generalized linear models via coordinate descent. Journal of Statistical Software, 33:1-122, 2010.

[14] I. Guyon, J. Weston, S. Barnhill, and V. Vapnik. Gene selection for cancer classification using support vector machines. Machine learning, 46(1-3):389-422, 2002.

[15] B. Kemper and G. von Bally. Digital holographic microscopy for live cell applications and technical inspection. Appl. Opt., 47(4):A52-A61, 2008.

[16] H. T. Ma and R. Y. Poon. Synchronization of HeLa Cells. In G. Banfalvi, editor, Cell Cycle Synchronization, volume 761, pages 151-161. Humana Press, Totowa, NJ, 2011.

[17] J. Moreau. Proximite et dualite dans un espace hilbertien. Bull. Soc.Math. France., 93, pages 273-299, 1965.

[18] H.-S. Pam Park and C.-H. Jun. A simple and fast algorithm for k-medoids clustering. expert systems with applications. Expert Systems with Applications, pages 3336-3341, 2009.

[19] J. Primot and N. Guérineau. Extended hartmann test based on the pseudoguiding property of a hartmann mask completed by a phase chessboard. Appl. Opt., 39:5715-5720, 2000.

[20] M. Purschke, N. Rubio, K. D. Held, and R. W. Redmond. Phototoxicity of Hoechst 33342 in time-lapse fluorescence microscopy. Photochemical \& Photobiological Sciences, 9(12): $1634,2010$.

[21] W. F. Scherer. Studies on the propagation in vitro of poliomyelitis viruses: iv. Viral multiplication in a stable strain of human malignant epithelial cells (strain HeLa) derived from an epidermoid carcinoma of the cervix. Journal of Experimental Medicine, 97 (5):695-710, May 1953.

[22] M. B. Taylor. Topcat stil: Starlink table/votable processing software. Astronomical Data Analysis Software and Systems XIV ASP Conference Series, 347:29, december 2005. 